\title{
Intelligent Control and Target-controlled Infusion
}

\author{
Na Wang ${ }^{1}$, Jinguo Wang ${ }^{2}$ and Dechuan Liu',a \\ ${ }^{1}$ The First Hospital of Jilin University, Department of Anesthesiology, 71 Xinmin Street, Changchun, China \\ ${ }^{2}$ The First Hospital of Jilin University, Department of Urology, 71 Xinmin Street, Changchun, China
}

\begin{abstract}
With the development of science and technology, intelligent control system has been used more and more widely. Closed-loop target-controlled infusion system is a typical example. This great combination of medicine and intelligent control system, especially in the field of anesthesia, solves many clinical problems. This article mainly introduces some typical examples of closed-loop target-controlled infusion system.
\end{abstract}

\section{Intelligent control}

Intelligent control is a new and interdisciplinary subject developed on multiple subjects, such as artificial intelligence and automatic control. It displays an advanced stage in the development of automatic control and solves the control problem of complex systems which is difficult to be solved by traditional methods. It is at the forefront of control science and represents the latest progress in the development of automatic control science $[1,2]$.

In 1967, Leonde and Mendel first used the concept of "intelligent control" to apply techniques of memory and goal decomposition to improve their ability to deal with uncertainty. In the 1970's, the Chinese scientist professor Fujing Sun used artificial intelligence reasoning method for robot control and learning control system. He put forward the development of intelligent control for the first time. This emerging discipline summarizes the artificial intelligence from the cybernetics and adaptive, self-organizing, self-learning control. The intelligent control summarized as the combination of automatic control and artificial intelligence. In 1987, the international symposium on intelligent control jointly held by IEEE and computer society marked the official emergence of intelligent control as an international scientific stage [3]. The IEEE control systems society of intelligent control system is defined as intelligent control system which can learn, memorize and have a wide range of adaptive and self organization ability [4]. It is able to adapt to changing conditions in time, effectively deal with all kinds of information and reduce the uncertainty. It can find safe and reliable way to plan and produce the control action to the expected goal and good performance [5].

Intelligence is the common pursuit of all industries. Intelligent control has been developing rapidly since its emergence and has been widely used in industry, agriculture, service industry, military aviation, medical treatment and other fields. This paper mainly introduces the application of intelligent control in target-controlled infusion (TCI) [6].

\section{Target-controlled infusion}

Intravenous medications often use standard dose guidelines, and patient characteristics such as weight, height, age, gender, and additional biomarkers are often excluded, because these covariates have complex mathematical relationships with doses. Each drug accumulates in the tissue during drug delivery, an accumulation that often confuses the relationship between the infusion rate prescribed by the clinician and the drug concentration in the patient [7]

On the base of the principles of pharmacokinetics, the TCI system can calculate how much the drug has accumulated in the tissue during infusion, adjust the infusion rate to maintain a stable concentration of plasma or interested tissue (usually the brain), and be able to use the best models in the literature combined with the mathematical complexity of patient characteristics [8]. The defects of serious circulation fluctuation, dosage increase, unstable anesthesia depth, unexpected wakefulness, respiratory depression and so on, which are easily caused by single administration, as well as the defects such as increase of dosage and delay of awakening caused by continuous administration, were avoided [8].

\subsection{Definition and background of $\mathrm{TCl}$}

\subsubsection{Backgroud of TCI}

As early as 1950, Mayo et al. used electrocortical activity to automatically titrate for analgesic administration

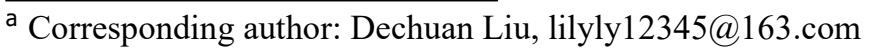


during abdominal surgery. Since then, many signals have been used to guide the automatic titration of different anesthetic drugs in various surgical environments. With the development of technology, the application of automation in the field of anesthesia is becoming more and more advanced, among which TCI technology is a great leap [9].

Since it was first listed in 1979, it has been developing and applied more and more widely. Approved TCI systems are available in more than 90 countries. More than 60,000 units of pumps have been installed and used to deliver product-based intravenous injections and anesthesia to millions of patients worldwide. It has become a mature technology in the recent 30 years since it was proposed [10]. It has been 20 years since it was first approved. The goal of all forms of medication is to achieve and maintain the treatment of drug effects while avoiding adverse effects. Target-controlled drug delivery mode has obvious advantages in safety, comfort, stability and economy [11].

\subsubsection{Definition of TCl}

TCI was originally applied in the field of anesthesia. It refers to that when injecting intravenous anesthetics, based on pharmacokinetics and pharmacodynamics, computers can calculate the amount of drug production in tissues during infusion, and adjust the infusion speed to maintain stable concentration in plasma or target tissue (brain usually) [12].

Traditional blood transfusions don't maintain stable concentrations of drugs in plasma or the brain during periods of sustained stimulation. The TCI system can rapidly titrate response when necessary and also maintain a stable concentration when appropriate. In addition, during the operation, the level of surgical stimulation changes rapidly, requiring accurate and rapid titration of the drug effect. Conventional infusion does not rapidly increase the concentration of the drug when the infusion needs to be enhanced, or rapidly decrease the concentration during the low stimulation period [13].

\subsection{Classification of TCI}

TCI system can be divided into two kinds of devices, including open loop and closed loop.

Open loop TCI has been widely used in clinical for many years. It is controlled by anesthesiologist according to patients' data (such as weight, age, etc.) and dose drug plasma concentration, combined with the vital signs such as electrocardiogram before and after medication, blood pressure, heart rate, blood oxygen content, respiratory function, the degree of muscle relaxation, depth of anesthesia monitoring data [14]. The anesthesiologist artificially adjusts the concentration of drug dose, so as to achieve the suitable concentration of anesthetic effect.

The research and development of closed loop was started in the 1980's, with the development of computer technology and EEG monitoring. Closed loop is combined with intelligent control system. Closed-loop TCI is constantly upgrading [15]. The main input part of closed-loop TCI is obtained by the real-time monitoring the vital signs of the plasma drug concentration or test index, and then it automatically adjusts and changes the dosing speed and the depth of anesthesia to meet the needs of real-time operation. Because the device is connected with feedback, according to the numerical index of clinical monitoring and compared with the set standard value, it can control the TCI data and the speed of the injection pump [16]. It can automatically reach the appropriate target control concentration and reduce the human error. An ideal closed-loop TCI system must include an anesthetic depth monitor which is prompt, accurate, free of interference and any use contraindication, capable of reflecting both sedation and analgesia, as well as a target-controlled infusion system without errors and bias and an ideal negative feedback system.

\section{Clinical application of intelligent control in $\mathrm{TCl}$}

\subsection{General anesthesia}

\subsubsection{General intravenous anesthesia}

A number of studies have shown that closed-loop control is more accurate than open-loop control, because it can deal with differences in pharmacokinetics among individuals, adapt to changes in shell stimulation, thus contributing to the rational use of anesthetic drugs and reducing the workload of anesthesiologists. At present, intelligent closed-loop control has been widely used in clinical applications, including cardiac surgery, pheochromocytoma surgery, gastrointestinal surgery and has had good clinical results [17].

Propofol was the most common drug choice in the infusion pump. BIS (bispectral index, referred to as the BIS) was the most common one in the intelligent feedback system. For example, figure 1, based on the design of intelligent control system, the pharmacokinetic model of human propofol, the drug delivery method of target controlled infusion of anesthesia, it proposes a system as the core and PID control algorithm for intelligent control of anesthesia depth. BIS can provide useful for individual patient anesthesia depth monitoring the trend of information, so desirable BIS as an indicator reflects the depth of anesthesia monitoring [18]. Also studies believe that the BIS while with the increase of depth of anesthesia and change the monotonous, closely associated with anesthesia hypnosis composition, but the response to surgical stimulation of prediction are less reliable, particularly in the use of different combination of hypnosis and analgesic drugs.

At present, the new generation of closed loop TCI often adopts the multi-phase feedback mechanism. At the same time, BIS and electromyogram are used for the clinical evaluation of the anesthesia depth and the nerve muscle block closed-loop anesthesia control system. There have also been numerous studies on closed loop TCI in special populations. For example in the closedloop TCI propofol for moderately severe left ventricular 
systolic dysfunction (ASA class III - IV) of anesthesia of critical care patients, compared with open loop, less dosage of propofol and BIS value more stable. Propofol closed-loop anesthesia is better than skilled manual control anesthesia in pediatric surgeries. Studies have shown that the amount of propofol in the closed loop group during induction and maintenance is basically the same as that in the manual control group in 7-14 pediatric anesthesia, but the time to maintain BIS value of $40 \sim 60$ is longer than that in the manual control group. The pharmacokinetic individual difference of propofol in children is relatively large, and the age is more obvious [19]. Therefore, it is of more clinical significance to use anesthesia depth monitoring to guide anesthesia medication.

\subsubsection{Inhalation of general anesthesia}

Compared with intravenous anesthesia, the inhalation anesthesia has many advantages. For patients with heart disease, the inhalation anesthetics has the advantages of ischemic preconditioning which can reduce myocardial enzyme increases and reduce the incidence of arrhythmia. Studies have shown that the use of isoflurane in cardiac surgery has higher survival rate compared with patients with propofol and fentanyl anesthesia after one-year follow up.

In 2011, Indian researchers Madhavan et al. successfully applied BIS to closed loop TCI system 23 for the first time, which can use propofol or isoflurane to regulate BIS in patients [8]. The control algorithm of propofol is based on the relationship between propofol infusion rate, plasma propofol concentration and BIS.The algorithm of inhaled anesthesia is based on the relationship between the BIS and the concentration of anaesthesia in the period of systematic development. Mainly includes the pentium or higher processor control algorithm of the personal computer (PC) provides users with interface, using RS232 serial port (Pilot-C, Fresenius, Brezins, France, etc.) infusion system, monitoring vital signs monitor and ventilation equipment with rising $\mathrm{CO}_{2}$ absorber and bellows (DatexOhmeda, software version 5.0, Helsinki, Finland) to provide constant volume ventilation, as shown in Figure 1. After open heart surgery of double-blind controlled clinical trial proves that the use of isoflurane closed-loop TCI (IDDS) patients compared with the traditional artificial, although the IDDS induction time is much longer, but artificial induced dose of propofol group is much higher, led to a decline in the BIS during induction of amplitude is bigger, the maximum BIS is similar in the two groups after intubation [13]. During the maintenance phase, the consumption of isoflurane in the IDDS group was significantly lower than that of the artificial group. More importantly, compared with manual control during isoflurane management, the closed-loop system was able to keep BIS in the target range for much longer (judged by lower MDPE) and the system performance was more accurate and less biased. Although the oscillations of the two groups were similar, the global rating IDDS group reflecting the overall performance of the system was significantly superior to the manual group.

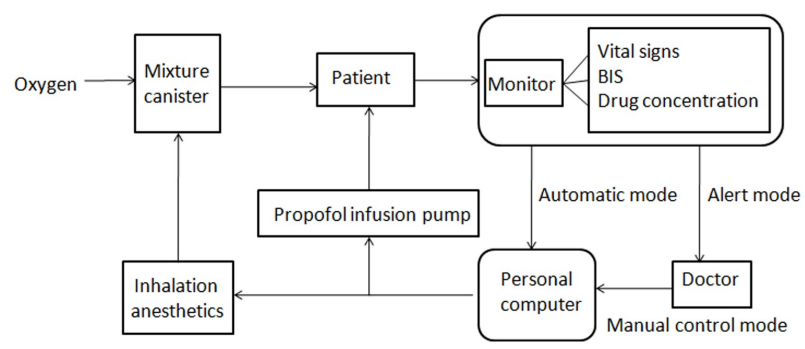

Figure 1. Apparatus for closed-loop anesthetics administration.

\subsection{Sedation and anesthesia}

Chiang et al. found in gastroenteroscopy and artificial regulation that patients given propofol TCI had faster recovery, more stable hemodynamics and respiratory system. The application of propofol to close loop anesthesia during digestive tract endoscopy in children can maintain reliable anesthesia depth and good spontaneous breathing.

\subsection{Diabetes treatment}

At present, TCI has been successfully applied in insulin treatment of diabetes, and has made remarkable progress in the control of patients' blood glucose and improvement of their quality of life. It is mainly a monitor that can monitor blood glucose in real time and feedback the information of blood glucose to the insulin infusion pump to guide the insulin infusion. A randomized controlled cross-trial of day-night closed insulin infusion in a large number of pregnant women with type 1 diabetes found that the infusion pump can effectively control their blood glucose levels, reducing the incidence of systemic and nocturnal hypoglycemia, while continuous monitoring of blood glucose in pregnant women with diabetes can reduce neonatal complications [17].

\subsection{Other aspects}

There have also been studies using TCI for antibiotic treatment, a closed-loop system of precise vancomycin delivery consisting of closed-loop controllers, subcutaneous sensors and intravenous pumps, potentially improving infection outcomes in non-critical populations.

\section{Summary and outlook}

At present, the closed-loop TCI system applied to TCI by intelligent control has been more and more widely used in clinical practice, and has played an increasingly important role in solving one medical problem after another. Of course, the system is not at the top level at present and still needs to be improved continuously, which is also one of our future efforts. 


\section{References}

1. Leondes CT, Mendel JM. Artiflcial intelligent control. Technical Report 4336, McDonnell-Douglas Astronautics Corporation, USA, 1967.

2. $\mathrm{Fu} \mathrm{KS}$. Learning control systems and intelligent control systems: an intersection of artiflcial intelligence and automatic control. IEEE Transactions on Automatic Control, 1971, 16(1): 7072.

3. Mayo CW, Bickford RG, Faulconer A Jr. Electroencephalographically controlled anesthesia in abdominal surgery. J Am Med Assoc, 1950; 144: 1081-3.

4. Absalom AR, Glen JI, Zwart GJ. Target-Controlled Infusion: A Mature Technology. Anesth Analg. 2016; 122(1): 70-78.

5. Hu C, Horstman DJ, Shafer SL. Variability of targetcontrolled infusion is less than the variability after bolus injection. Anesthesiology. 2005; 102: 639-45.

6. Struys M, de Smet T. Principles of drug actions: target-controlled infusions and closed-loop administration. In: Evers AS, Maze M, Kharasch ED, eds. Anesthetic Pharmacology: Basic principles and Clinical Practice. Cambridge, UK: Cambridge University Press, 2011:103-122

7. Struys MM, De Smet T, Glen JI. The History of Target-Controlled Infusion. Anesth. Analg. 2016; 122(1): 56-68.

8. C Yang, N Chen. Advances in Target Controlled Infusion for Anesthesia. Chinese Medical Equipment Journal. 2012; 33(2): 89-91

9. Kenny GN, Mantzaridis H. Closed-loop control of propofol anaesthesia. Br J Anaesth. 1999; 2: 223-228.

10. Biswas I, Mathew PJ, Singh RS. Evaluation of closed-loop anesthesia delivery for propofol anesthesia in pediatric cardiac surgery. Paediatr Anaesth. 2013; 23: 1145-1152.

11. Hemmerling TM, Arbeid E, Wehbe M. Evaluation of a novel closed-loop total intravenous anaesthesia drug delivery system: a randomized controlled trial. Br J Anaesth. 2013; 110: 1031-1039.

12. Liu N, Le Guen M, Benabbes-Lambert F,et al. Feasibility of closed-loop titration of propofol and remifentanil guided by the spectral M-Entropy monitor. Anesthesiology. 2012; 116: 286-95.

13. J Guo, GS Yang, XD Xue. Application of narcotrend-guided propofol closed-loop targetcontrolled infusion in the gastrointestinal tumor operation. Chinese Journal of New Drugs. 2018, 27(5): 540-548.

14. Coetzee JF, Glen JB, Wium CA, Boshoff L. Pharmacokinetic model selection for target controlled infusions of propofol: assessment of three parameter sets. Anesthesiology. 1995; 82: 1328e45.

15. Dussaussoy C, Peres M, Jaoul V. Automated titration of propofol and remifentanil decreases the anesthesiologist's workload during vascular or thoracic surgery: a randomized prospective study. J Clin Monit Comput. 2014; 28: 35-40.

16. Liu Y, Li M, Yang D, Zhang X, Wu A, Yao S. Closed-Loop Control Better than OpenLoop Control of Profofol TCI Guided by BIS: A Randomized, Controlled, Multicenter Clinical Trial to Evaluate the CONCERT-CL Closed-Loop System. Plos One. 2015; 10(4): e0123862.

17. Lee AKY, Kong AYH, Kong CF. Performance of TCI Propofol Using the Schnider Model for Cardiac Surgery on Cardiopulmonary Bypass-A Pilot Study. J Cardiothorac Vasc Anesth 2018; 32 (2): 723-30.

18. Hegde HV, Puri GD, Kumar B, Behera A. Bispectral index guided closed-loop anaesthesia delivery system (CLADS) in pheochromocytoma. J Clin Monit Comput. 2009; 23: 189-96.

19. DONG Liang, ZHANG Xing-an, XIONG Dongsheng1. The Design of Anaesthesia Depth Intelligent Control System Based on TCI. Chinese Journal of Medical Physics. 2013; 30(2): 4052-6. 